\title{
Type 2 diabetes and risk of hospital admission or death for chronic liver diseases.
}

Sarah H Wild ${ }^{1}$, Joanne R Morling ${ }^{1}$, David A McAllister ${ }^{1}$, Jan Kerssens ${ }^{2}$, Colin Fischbacher ${ }^{2}$, Julie Parkes ${ }^{3}$, Paul J Roderick ${ }^{4}$, Naveed Sattar ${ }^{5}$, Christopher D Byrne 6,7 on behalf of the Scottish and Southampton Diabetes and Liver Disease Group and the Scottish Diabetes Research Network Epidemiology Group

${ }^{1}$ Centre for Population Health Sciences, Usher Institute of Population Health Sciences and Informatics, University of Edinburgh, UK

2 Information Services Division, National Health Service National Services Scotland, Edinburgh, UK

${ }^{3}$ Faculty of Medicine, University of Southampton, UK

${ }^{4}$ Primary Care and Population Sciences, University of Southampton, UK

${ }^{5}$ British Heart Foundation Centre for Cardiovascular Science, University of Glasgow, UK

${ }^{6}$ Nutrition and Metabolism, Faculty of Medicine, University of Southampton, UK

${ }^{7}$ NIHR Southampton Biomedical Research Centre, University Hospital Southampton, UK

Further members of the Scottish and Southampton Diabetes and Liver Disease Group (John Dillon, Peter Hayes, David Macfarlane, John Petrie, Nick Sheron) and the Scottish Diabetes Research Network epidemiology group (Helen Colhoun, Robert Lindsay, Graham Leese, Helen Looker, John McKnight, Rory McCrimmon, Sam Philip)

\section{Corresponding author:}

Professor Sarah Wild, Centre for Population Health Sciences, Usher Institute of Population Health Sciences and Informatics, University of Edinburgh, Teviot Place, Edinburgh, EH8 9AG, UK. Tel: +44 1316511630 Fax: +44 131650 6868. Email: sarah.wild@ed.ac.uk.

Word count including abstract, references, tables and figure legends: 5642

Number of figures and tables: 2 figures and 5 tables 


\section{List of abbreviations in order of appearance:}

T2DM - type 2 diabetes

CLD - chronic liver disease

ALD - alcoholic liver disease

NAFLD - non-alcoholic fatty liver disease

SES - socio-economic status

$\mathrm{RR}$ - rate ratio

HCC - hepatocellular carcinoma

$\mathrm{NASH}$ - non-alcoholic steatohepatitis

SIMD -Scottish Index of Multiple Deprivation

T1DM - type 1 diabetes

BMI -body mass index

SCI-DC - Scottish Care Information - Diabetes Collaboration

Keywords: diabetes; liver disease; cohort

Conflict of interest: none

Financial support: this work was supported by funding from the Scottish Government through the Scottish Diabetes Group

Author contributions: JRM and CDB performed the literature search, SHW, JRM, DAM, JK, CF, NS and CDB designed the study and the analysis plan, data were acquired as part of routine clinical care, JK prepared the data, SHW analysed the data and prepared the figures, SHW and CDB wrote the first draft of the manuscript and all authors contributed to data interpretation, critically reviewed the manuscript for important intellectual content and approved the final version. The work was performed on behalf of the Scottish and Southampton Diabetes and Liver Disease Group and the Scottish Diabetes Research Network Epidemiology Group. 


\section{Abstract}

Background \& Aims: The impact of type 2 diabetes (T2DM) on hospital admissions and deaths due to common chronic liver diseases (CLDs) is uncertain. Our aim was to investigate associations between T2DM and CLDs in a national retrospective cohort study and to investigate the role of sex and socio-economic status (SES). Methods: We used International Classification of Disease codes to identify incident alcoholic liver disease (ALD), autoimmune liver disease, haemochromatosis, hepatocellular carcinoma, non-alcoholic fatty liver disease (NAFLD) and viral liver disease from linked diabetes, hospital, cancer and death records for people of 40-89 years of age in Scotland 2004-2013. We used quasi Poisson regression to estimate rate ratios $(R R)$.

Results: There were 6667 and 33624 first mentions of CLD in hospital, cancer and death records over $\sim 1.8$ and 24 million person years in people with and without T2DM, respectively. The most common liver disease was ALD among people without diabetes and was NAFLD among people with T2DM. Age adjusted RR for T2DM compared to the non-diabetic population (95\% confidence intervals) varied between 1.27 (1.04-1.55) for autoimmune liver disease and 5.36 (4.41-6.51) for NAFLD. RRs were lower for men than women and for more compared to less deprived populations for both ALD and NAFLD.

Conclusions: T2DM is associated with increased risk of hospital admission or death for all common CLDs and the strength of the association varies by type of CLD, sex and SES. Increasing prevalence of T2DM is likely to result in increasing burden of all CLDs.

Keywords: diabetes; liver disease; cohort 


\section{Introduction}

Mortality from liver disease in the United Kingdom (UK) has increased by $400 \%$ since the 1970s, in contrast to reductions in mortality from cardiovascular disease and some cancers [1]. Similar increases in CLD mortality have also been observed in the United States (US)[2] although cirrhosis mortality has declined by $50-60 \%$ in France and Italy between 1980 and 2010[3]. Incidence and prevalence of common chronic liver diseases (CLDs) including alcohol-related liver disease (ALD) and obesity-related non-alcoholic fatty liver disease (NAFLD) and their complications have increased in the UK and US [4, 5]. Age-standardised incidence and mortality from hepatocellular carcinoma (HCC), a complication of many CLDs, have increased approximately two-fold in the UK in the last 40 years[4] and in other European countries (see http://globocan.iarc.fr/old/FactSheets/cancers/liver-new.asp). It is well known that type 2 diabetes (T2DM) is associated with increased risk of the whole spectrum of NAFLD and its complications including non-alcoholic steatohepatitis (NASH), cirrhosis and HCC[6-9], but the effect of T2DM on other common types of CLD is less clear.

Estimating the burden of ill health attributable to CLDs is difficult due to the lack of accurate, non-invasive diagnostic tests that can be applied within population-based studies. The available tests have been predominantly validated in high prevalence (secondary care) populations and may be less accurate in the wider population. Routine healthcare data obtained from hospital admission, cancer registration and death records provides a source of readily available data that have been used previously to assess the burden of serious gastro-intestinal disease in the US[5]. Although hospital admission and cancer registration data are likely to underestimate 
prevalence of disease they provide valuable information for health care planning and an estimate of the burden of serious liver disease.

Increasing global prevalence of T2DM and the inverse influence of socio-economic status (SES) on T2DM prevalence in high income countries are well established [10, 11]. The inverse relationship between SES and ALD has also been described in several populations[12,13] and the incidence of HCC in England between 1990 and 2009 was higher among people living in materially deprived areas than among people living in less deprived areas[14]. However, it is not known whether SES influences the association between T2DM and CLDs, as it does for T2DM and cardiovascular disease [15]. The need for further research on the contribution of SES to disparities in incidence, treatment and outcomes of liver disease has previously been identified[16, 17].

The aim of this retrospective cohort study in a national population of approximately five million people was: a) to describe numbers and rates of first record of CLD hospital admission or death for people with and without T2DM and, b) to investigate associations between T2DM and CLD hospital admissions and deaths, establishing whether associations differed by sex or SES.

\section{Methods}

\section{Datasets}

We used data from the population-based Scottish diabetes register linked to national hospital, cancer and death records in a retrospective cohort study design. The Scottish diabetes register is derived from primary and secondary care records for 
people with diabetes diagnosed in normal clinical practice using blood glucose measurements and/or $\mathrm{HbA} 1 \mathrm{c}$ with coverage of $>99 \%$ since $2004[18]$ the start of the study period. Validation of the diabetes register for clinical practice occurs through responses to annual invitations to diabetic retinopathy screening. For example, in 2010 191,571 individuals were invited for screening of whom $2 \%$ were suspended from the screening programme due to the person not having diabetes (see http://www.scidiabetes.scot.nhs.uk/project-overview/). In addition, validation of the research extract, performed against hospital admissions mentioning diabetes[18] or against sulphonylurea prescriptions (unpublished data) suggests that over $99 \%$ of people with diagnosed diabetes in Scotland are included in both clinical and research databases.

\section{Definition of CLD}

CLD was identified from the first mention in any position (primary/underlying or secondary diagnosis or cause of death) in national hospital in-patient, cancer and mortality records and grouped into categories using codes from the ninth (ICD-9) and tenth [ICD-10] International Classification of Diseases revisions as follows:

- ALD: (571.0), (571.2), (571.3), [K70];

- autoimmune hepatitis and primary biliary cirrhosis: (571.4), (571.6), [K75.4, K74.3];

- hemochromatosis: (275.0) [E83.1];

- hepatocellular carcinoma (HCC): (155.0); [C22.0];

- NAFLD: a category incorporating the following ICD-defined categories: other chronic non-alcoholic liver disease: (571.8), non-alcoholic fatty liver disease: [K76.0], non-alcoholic steatohepatitis: [K75.8], cirrhosis (571.5) [K74.6], hepatic fibrosis or sclerosis or fibrosis with sclerosis (571.9) [K74.0, K74.1, 
K74.2] or portal hypertension: (572.3) [K76.6] that was not attributed to any of the other liver disease categories.

- viral hepatitis: (D070.3), (D070.5), (D070.9); [B16, B17, B18];

The study size was determined by the number of incident CLDs occurring in the national population in the study time period which ended in December 2013, based on the most recent data available to the research team.

An area-based measure of SES is assigned to residents of Scotland on the basis of where they live using the Scottish Index of Multiple Deprivation (SIMD) (for more information see http://www.scotland.gov.uk/Topics/Statistics/SIMD). SIMD 2009 combines 31 indicators across seven domains: income/employment, health, education, housing, geographic access, and crime. The index is generated from a weighted sum of the seven domain scores for each area defined by postcodes which contains a median of 769 people. Quintiles of the index were used to define approximately equal fifths of the distribution at a national level, and Q1 and Q5 were used to identify the most and least deprived quintiles (or fifths) respectively.

\section{Study design and data analysis}

We used a dynamic population-based retrospective study design using aggregated data for the total population of Scotland and individual level data on people with diabetes. Inclusion criteria for estimation of the person-years denominator were being aged 40 to 89 years in Scotland between 2004 and 2013. The only exclusion criteria were the approximately $1 \%$ of hospital admissions or death records with missing SIMD data and the events and person-years for people with type 1 diabetes. 
For each CLD group, with the exception of NAFLD, the first recorded event was identified independently (so, for example, a record of hemochromatosis followed by a subsequent record of viral hepatitis contributes to event counts and person-years denominator for both disease groups). Event counts and person-years at risk for NAFLD were identified by excluding records with any mention of ALD, viral disease, hemochromatosis or auto-immune liver disease. Records that mentioned HCC and NAFLD and no other specific CLD were counted in both HCC and NAFLD groups.

Routine national healthcare data were used to identify CLD events occurring between January 12004 and December 312013 for adults aged 40-89 years. For each CLD, people with prevalent disease prior to January 2004 defined by the presence of a relevant disease code in hospital or cancer records in the previous 10 years were excluded. Follow-up time was censored for each CLD at first event or death from any cause. We included deaths from CLDs and HCC where they represented the record of CLD or HCC and there was no previous hospital or cancer record of CLD or HCC. Numbers of CLD events, person-years at risk and rates were estimated by diabetes status, age, sex, and SIMD quintile.

Mid-year population estimates for Scotland by age, sex, calendar year, and SIMD quintile for the relevant years were obtained from National Records of Scotland. Data for the non-diabetic population were identified by subtracting numbers of incident events and person-years at risk for the population of people with type 1 diabetes (T1DM) and T2DM from numbers of CLD events and person-years at risk calculated from mid-year populations for the whole of Scotland. Type of diabetes was based on clinical records and a validation algorithm that used information on age at diagnosis 
of diabetes with additional validation using dates and type of diabetes treatment, dates of first $\mathrm{HbA} 1 \mathrm{c}>6.5 \%(48 \mathrm{mmol} / \mathrm{mol})$ and invitation to diabetic retinopathy screening[19].

Absolute incidence rates were used to describe patterns of different CLDs in the study population, with direct standardisation for age, stratification by sex and, where appropriate, for SIMD quintile, using the 2007 mid-year population of Scotland as the standard. Rate ratios (RR) for people with T2DM compared to people without diabetes were estimated using quasi Poisson regression modelling to control for potential confounding by age and to allow for the over-dispersed nature of the distribution of the data. Likelihood ratio tests were used to test for interactions between T2DM and sex and T2DM and SES. Analyses were stratified by sex and also for SES where appropriate. Exploratory analyses were performed among people with type 1 diabetes.

Sensitivity analyses were performed to identify whether the associations with T2DM altered if the following alternative outcome definitions were used

1. primary diagnoses/ underlying cause of death of ALD and NAFLD (excluding mention of ALD or NAFLD in secondary positions in hospital and death records)

2. hospital admission records mentioning ALD and NAFLD in any position (excluding deaths with no prior record of hospital admission)

3. hospital and death records mentioning chronic non-alcoholic liver disease: (ICD-9 571.8), non-alcoholic fatty liver disease: [ICD-10 K76.0], and nonalcoholic steatohepatitis: [ICD-10 K75.8] in any position (excluding 
cirrhosis, hepatic fibrosis/sclerosis and portal hypertension of unspecified cause).

Approval for the creation and analysis of the linked dataset containing no personal identifying information was obtained from the Scottish Care Information - Diabetes Collaboration (SCl-DC) steering committee, the Scottish multi-centre research ethics committee (reference number 11/AL/0225), the Privacy Advisory Committee of National Health Service National Services Scotland and Caldicott guardians. Stata version 13 (College Station, Texas) was used for the analyses.

\section{Results}

During approximately 26 million years of follow-up in total there were a total of 40291 first records of CLD events identified from hospital or death records in people aged 40 to 89 years in Scotland in the decade between 2004 and 2013. There were 6667 events in people with T2DM during 1.8 million person-years of follow-up. Approximately $61 \%$ of CLD records were identified in men regardless of diabetes status whereas men represent $54 \%$ of the population of people with T2DM in Scotland over 40 years of age and $47 \%$ of the whole population in this age group in 2008. The majority (97.4\%) of people with a record of a specific incident CLD had only one of ALD, auto-immune liver disease, hemochromatosis, HCC, or viral liver disease mentioned in their records. 
Table 1 shows the numbers of first records of CLDs and age-standardised rates by diabetes status, sex and type of liver disease. The majority of outcomes were identified from hospital records or cancer registrations as shown in table 2 . The highest CLD rates were for ALD for people without diabetes and for NAFLD for people with T2DM. The range of rates across SIMD quintiles is given for ALD and NAFLD because of the statistically significant variation in incidence by SES (see figures 1 and 2). People with T2DM were older than those without diabetes at the time of first CLD event and a large proportion of CLD events occurred among people of working age (data not shown but available from authors).

Age-standardised rates by sex, diabetes status and SES for ALD and NAFLD are shown in figure 1 and figure 2 respectively and illustrate higher rates in more compared to less deprived populations and a more marked socio-economic gradient for ALD than NAFLD. Age-adjusted RRs for people with T2DM compared to people without diabetes for ALD and NAFLD varied with sex and SES (Table 3). We identified a statistically significant interaction between diabetes status and SES for ALD and NAFLD but there was no evidence of similar interaction for other CLDs. RRs for ALD and NAFLD were lower among more deprived than among less deprived populations. There was a statistically significant interaction between T2DM and sex for NAFLD as a consequence of markedly higher relative risk of NAFLD among women than men $(p<0.0001)$, but no evidence for an interaction between T2DM and sex for ALD ( $p=0.870)$.

Table 4 shows adjusted RRs for each CLD for people with T2DM compared to people without diabetes. Further adjustment for SES gave similar estimates of RRs 
to age-adjusted results suggesting minimal confounding by SES. T2DM was associated with statistically significantly higher risk of all types of CLD apart from for

viral liver disease in men. The highest RRs associated with T2DM were for HCC and NAFLD. Summary estimates of RRs are shown for ALD and NAFLD to allow comparison with RRs for other liver diseases although the interaction illustrated in the figures and reported in table 3 should be noted. The sensitivity analyses for different outcome measures for ALD and NAFLD are reported in Table 5 and show similar RRs for the associations with T2DM as for those reported for the original analyses.

\section{Discussion}

This national retrospective cohort study of a contemporary Scottish population studied over a decade between 2004 and 2013. The absolute rates and relative risks associated with T2DM varied markedly by type of CLD. The strength of the associations between T2DM and CLD varied with sex for NAFLD and with SES for both ALD and NAFLD. In keeping with previous data, T2DM was associated with particularly high relative risks of HCC[20] and NAFLD[1], but we report for the first time that there is also increased risk associated with T2DM for all other types of CLD including ALD. There was only minimal confounding of the association between T2DM and CLD by SES but there was evidence of interaction between T2DM and SES for ALD and NAFLD. The absolute risk and the relative risk of NAFLD associated with T2DM were statistically significantly higher for women than men. 
Socio-economic deprivation was associated with higher incidence of ALD regardless of diabetes status.

These findings have important implications for prevention and management of CLD and for the planning and the costs of health services, given the increasing prevalence of T2DM and the considerable cost of hospital admissions. Health care costs for patients with severe complications of CLDs are particularly high as illustrated by recent data for patients with various stages of liver disease related to hepatitis $C$ from the US[21]. Costs per-patient-per-month over a mean of 634 days of follow-up between 2002 and 2010 amounted to $\$ 1,420, \$ 1,870$ and $\$ 4,931$ for people with non-cirrhotic liver disease, compensated cirrhosis and end-stage liver disease respectively ( $\$ 1=0.93$ Euros November 2015$)$. The annual costs to the National Health Service in England in 2007 for cirrhosis and liver cancer due to alcohol consumption alone was estimated to be $£ 566$ million[22] ( $£ 1=1.43$ Euros November 2015).

The associations between T2DM and CLDs by sex and SES are likely to reflect differences in distributions of risk factors for both CLDs and for T2DM including obesity, fat distribution and alcohol intake. For example, we have previously reported higher mean body mass index (BMI) among women than men for a given age at diagnosis of T2DM and higher prevalence of obesity $(\mathrm{BMI} \geq 30 \mathrm{~kg} / \mathrm{m} 2)$ in the most deprived compared to the least deprived fifths of the population around the time of diagnosis of diabetes (54\% compared to $44 \%$ )[23] In the population based Scottish Health Survey 2003, obesity prevalence was $34 \%$ in the most deprived quintile and $21 \%$ in the least deprived quintile[24]. Obesity and modest alcohol intake have 
previously been found to interact to further increase risk of cirrhosis in a Scottish population[25]. The higher prevalence of obesity in deprived populations is therefore likely to contribute to higher prevalence of cirrhosis regardless of diabetes status and underlying alcoholic or non-alcoholic CLD etiology.

As both CLD and T2DM may have long asymptomatic phases prior to diagnosis, it is difficult to identify which disease develops first and to identify the true incidence of either disease and whether the association with CLDs and subsequent cirrhosis is bidirectional. Detailed characterisation in cohort studies with repeated measurements is required to determine whether individual CLDs with or without cirrhosis are associated with increased risk of developing subsequent T2DM. For example, hepatic steatosis identified by ultrasound has been identified as a risk factor for subsequent incident diabetes compared to people without hepatic steatosis[6], and resolution of hepatic steatosis is associated with reduction of risk of incident diabetes compared to people with persistent hepatic steatosis[7]. Indeed, it is estimated up to $70 \%$ of patients with T2DM have hepatic steatosis and increasing evidence indicates that increases in liver fat contributes to the pathogenesis of T2DM [26].

The strengths of our study include the very large population-based nature of the electronic record of diagnosed diabetes that captures data for $>99 \%$ of the population of Scotland, validation of type of diabetes, and the availability of linkage to quality-assured hospital admission, cancer registration and mortality data for the whole population (for more information, see http://www.isdscotland.org/Productsand-Services/Data-Quality/Assessments/). 
We identified first hospital admission and deaths in people in Scotland mentioning CLD whether or not this was recorded as the primary diagnosis in hospital or underlying cause of death in death records for our primary outcome measures. We believe that this is the most appropriate approach for identifying CLD from routine health care data at present because co-morbidities such as cardiovascular disease $[27,28]$ may be coded as the primary diagnosis in hospital records or underlying cause of death in people with CLDs. Accuracy and completeness of recording of secondary conditions on health records may differ by diabetes status which could introduce bias in either direction. People with diabetes may be more likely to have liver disease diagnosed as a consequence of larger numbers of health service contacts and are also be more likely to be admitted to hospital than people without diabetes but other co-morbidities may be recorded in preference to CLD among people with diabetes. Our sensitivity analyses identified that the associations with T2DM were similar when the outcomes were limited to primary diagnosis in hospital records or underlying cause of death suggesting that bias in recording did not influence our original findings. We are not able to describe the population-based incidence of each CLD in people with and without diabetes, because our measure of data capture only identifies non-fatal diagnoses of CLD recorded during a hospital admission. Consequently our data capture omits diagnoses established solely in primary or ambulatory care that are likely to represent less severe forms of each liver disease. Our identification of the first CLD event and the interpretations of the associations with diabetes are based on the assumption that most of the previous events can be identified in Scottish records and that proportions of events occurring outside Scotland in people with and without diabetes are similar. 
Validation of coding of CLD and its complications is clearly required to facilitate population-based research. For example, the use of the narrow National Center for Health Statistics definition of CLD which excludes viral hepatitis and hepatobiliary cancer has been reported to systematically under-estimate liver-related mortality in the United States[30]. We found similar rate ratios associated with T2DM whether or not we included cirrhosis, hepatic fibrosis/ sclerosis and portal hypertension in the NAFLD outcome but it is not clear without validation whether it would be appropriate to include these outcomes in estimates of NAFLD resource use. Validated coding of CLD would also improve future research including cohort studies of the association between different CLDs and HCC, cirrhosis, transplantation and mortality in people with and without diabetes and the investigation of effects of different treatments for diabetes on CLD outcomes.

There is potential for mis-classification of ALD and NAFLD which will co-exist in a large proportion of people in populations where alcohol consumption and obesity are common. The approach we have taken means that records that include both diagnoses are identified as ALD, with consequent under-counting of records of NAFLD. It is likely therefore that we have under-estimated the strength of the association between T2DM and hospital admissions and deaths for people with a record of NAFLD. There is an argument for combining CLD into a single category but we elected not to do this because of the different patterns of association between T2DM and different CLDs.

The use of an area-based measure of deprivation, rather than an individual-based measure of SES is a potential limitation of the study. However, the small average 
population size of each area used to create the SIMD (median $<800$ people) means that in general it performs well as a measure of material deprivation. The inclusion of the health domain in SIMD does not seem to have a major effect on the pattern of health inequalities by SIMD[29] The predominantly white ethnic distribution of the Scottish population means that it is not feasible to investigate ethnic differences in CLD incidence. We are not able to describe the population-based incidence of each CLD in people with and without diabetes because we were not able to identify diagnoses of CLD established in primary or ambulatory care.

Key limitations of this analysis are that records of lifetime alcohol intake and measures of diet and physical activity are not available in this database and BMI is only available for people with diabetes. We were therefore not able to estimate what proportion of the apparent effect of T2DM could be explained by the association with obesity. The study had limited power to detect statistically significant interactions for less common liver diseases than ALD and NAFLD and to investigate the association between T1DM and CLD. However, based on 115 ALD events among people with T1DM in our population, we found age, sex and SES adjusted RRs (95\% confidence intervals) associated with type 1 diabetes of 1.09 (0.69-1.72). There was a statistically significant interaction between type 1 diabetes and sex for the association with NAFLD, with age and SES adjusted RR of 3.03 (2.68-3.43) for men and 5.36 (4.41-6.51) for women based on 75 events in men and 78 in women.

\section{Conclusions}


These findings show that people with T2DM in Scotland are at higher risk of first hospital admission, hepato-cellular cancer registration or death from all types of common chronic liver diseases, compared to people without diabetes. Adjustment for an area-based measure of SES only modestly attenuated the contribution of T2DM to hospital admission or death from CLD. SES modified the associations between T2DM and ALD and NAFLD and sex modified the association between T2DM and NAFLD. We suggest that there may be a role for targeted case finding of CLD and appropriate intervention in high risk populations including people with T2DM. The increasing global prevalence of T2DM can be expected to result in an increasing burden of all CLDs.

\section{Acknowledgements}

This work was supported by funding from the Scottish Government through the Scottish Diabetes Group. Diabetes data for Scotland are available for analysis thanks to numerous healthcare staff who enter the data and people and organisations (people with diabetes, the Scottish Care Information - Diabetes Collaboration [SCI-DC] Steering Group, the Scottish Diabetes Group, the Scottish Diabetes Survey Group, the managed clinical network managers and staff in each Health Board) involved in providing data, setting up, maintaining and overseeing SCI-DC. CDB is supported in part by the Southampton National Institute for Health Research Biomedical Research Centre. 


\section{Figure Legends}

Figure 1. Age-standardised incidence of hospital admissions and deaths for alcoholic liver disease per 100000 person years by sex, diabetes status (circles represent data for people without diabetes, squares for people with T2DM) and quintile of socioeconomic status (SES, where 1 indicates the most deprived quintile)

Figure 2. Age-standardised incidence of hospital admissions and deaths for nonalcoholic fatty liver disease per 100000 person years by sex, diabetes status (circles represent data for people without diabetes, squares for people with T2DM) and quintile of socio-economic status (SES, where 1 indicates the most deprived quintile) 


\section{Reference List}

[1] Williams R, Aspinall R, Bellis M, Camps-Walsh G, Cramp M, Dhawan A, et al. Addressing liver disease in the UK: a blueprint for attaining excellence in health care and reducing premature mortality from lifestyle issues of excess consumption of alcohol, obesity, and viral hepatitis. Lancet 2014 Nov 29;384(9958):1953-1997.

[2] Kim Y, Ejaz A, Tayal A, Spolverato G, Bridges JF, Anders RA, et al. Temporal trends in population-based death rates associated with chronic liver disease and liver cancer in the United States over the last 30 years. Cancer 2014 Oct 1;120(19):30583065.

[3] Mokdad AA, Lopez AD, Shahraz S, Lozano R, Mokdad AH, Stanaway J, et al. Liver cirrhosis mortality in 187 countries between 1980 and 2010: a systematic analysis. BMC Med 2014;12:145.

[4] Ladep NG, Khan SA, Crossey MM, Thillainayagam AV, Taylor-Robinson SD, Toledano MB. Incidence and mortality of primary liver cancer in England and Wales: changing patterns and ethnic variations. World J Gastroenterol 2014 Feb $14 ; 20(6): 1544-1553$.

[5] Peery AF, Dellon ES, Lund J, Crockett SD, McGowan CE, Bulsiewicz WJ, et al. Burden of gastrointestinal disease in the United States: 2012 update.

Gastroenterology 2012 Nov;143(5):1179-1187.

[6] Sung KC, Jeong WS, Wild SH, Byrne CD. Combined influence of insulin resistance, overweight/obesity, and fatty liver as risk factors for type 2 diabetes. Diabetes Care 2012 Apr;35(4):717-722.

[7] Sung KC, Wild SH, Byrne CD. Resolution of fatty liver and risk of incident diabetes. J Clin Endocrinol Metab 2013 Sep;98(9):3637-3643.

[8] Wang C, Wang X, Gong G, Ben Q, Qiu W, Chen Y, et al. Increased risk of hepatocellular carcinoma in patients with diabetes mellitus: a systematic review and meta-analysis of cohort studies. Int J Cancer 2012 Apr 1;130(7):1639-1648.

[9] Walker JJ, Brewster DH, Colhoun HM, Fischbacher CM, Leese GP, Lindsay RS, et al. Type 2 diabetes, socioeconomic status and risk of cancer in Scotland 20012007. Diabetologia 2013 Aug;56(8):1712-1715.

[10] Guariguata L, Whiting DR, Hambleton I, Beagley J, Linnenkamp U, Shaw JE. Global estimates of diabetes prevalence for 2013 and projections for 2035. Diabetes Res Clin Pract 2014 Feb;103(2):137-149.

[11] Walker JJ, Livingstone SJ, Colhoun HM, Lindsay RS, McKnight JA, Morris AD, et al. Effect of socioeconomic status on mortality among people with type 2 diabetes: a study from the Scottish Diabetes Research Network Epidemiology Group. Diabetes Care 2011 May;34(5):1127-1132. 
[12] Chartier K, Caetano R. Ethnicity and health disparities in alcohol research. Alcohol Res Health 2010;33(1-2):152-160.

[13] Makela P. Alcohol-related mortality as a function of socio-economic status. Addiction 1999 Jun;94(6):867-886.

[14] Konfortion J, Coupland VH, Kocher HM, Allum W, Grocock MJ, Jack RH. Time and deprivation trends in incidence of primary liver cancer subtypes in England. $J$ Eval Clin Pract 2014 Jun 5.

[15] Jackson CA, Jones NR, Walker JJ, Fischbacher CM, Colhoun HM, Leese GP, et al. Area-based socioeconomic status, type 2 diabetes and cardiovascular mortality in Scotland. Diabetologia 2012 Nov;55(11):2938-2945.

[16] Guy J, Yee HF, Jr. Health disparities in liver disease: Time to take notice and take action. Hepatology 2009 Jul;50(1):309-313.

[17] Nguyen GC, Thuluvath PJ. Racial disparity in liver disease: Biological, cultural, or socioeconomic factors. Hepatology 2008 Mar;47(3):1058-1066.

[18] Anwar H, Fischbacher CM, Leese GP, Lindsay RS, McKnight JA, Wild SH. Assessment of the under-reporting of diabetes in hospital admission data: a study from the Scottish Diabetes Research Network Epidemiology Group. Diabet Med 2011 Dec;28(12):1514-1519.

[19] Livingstone SJ, Looker HC, Hothersall EJ, Wild SH, Lindsay RS, Chalmers J, et al. Risk of cardiovascular disease and total mortality in adults with type 1 diabetes: Scottish registry linkage study. PLoS Med 2012;9(10):e1001321.

[20] Yang WS, Va P, Bray F, Gao S, Gao J, Li HL, et al. The role of pre-existing diabetes mellitus on hepatocellular carcinoma occurrence and prognosis: a metaanalysis of prospective cohort studies. PLoS One 2011;6(12):e27326.

[21] Gordon SC, Pockros PJ, Terrault NA, Hoop RS, Buikema A, Nerenz D, et al. Impact of disease severity on healthcare costs in patients with chronic hepatitis $\mathrm{C}$ (CHC) virus infection. Hepatology 2012 Nov;56(5):1651-1660.

[22] Scarborough P, Bhatnagar P, Wickramasinghe KK, Allender S, Foster C, Rayner M. The economic burden of ill health due to diet, physical inactivity, smoking, alcohol and obesity in the UK: an update to 2006-07 NHS costs. J Public Health (Oxf) $2011 \mathrm{Dec} ; 33(4): 527-535$.

[23] Logue J, Walker JJ, Leese G, Lindsay R, McKnight J, Morris A, et al. Association between BMI measured within a year after diagnosis of type 2 diabetes and mortality. Diabetes Care 2013 Apr;36(4):887-893.

[24] Lawder R, Harding O, Stockton D, Fischbacher C, Brewster DH, Chalmers J, et al. Is the Scottish population living dangerously? Prevalence of multiple risk factors: the Scottish Health Survey 2003. BMC Public Health 2010;10:330. 
[25] Hart CL, Morrison DS, Batty GD, Mitchell RJ, Davey Smith G. Effect of body mass index and alcohol consumption on liver disease: analysis of data from two prospective cohort studies. BMJ 2010 Mar 11;340:c1240.

[26] Sattar N, Gill JM. Type 2 diabetes as a disease of ectopic fat? BMC Med $2014 ; 12: 123$.

[27] Ekstedt M, Hagstrom H, Nasr P, Fredrikson M, Stal P, Kechagias S, et al. Fibrosis stage is the strongest predictor for disease-specific mortality in NAFLD after up to 33 years of follow-up. Hepatology 2014 Aug 14.

[28] Byrne CD, Targher G. NAFLD: a multisystem disease. J Hepatol 2015 Apr;62(1 Suppl):S47-S64.

[29] Adams J, White M. Removing the health domain from the Index of Multiple Deprivation 2004-effect on measured inequalities in census measure of health. $J$ Public Health (Oxf) 2006 Dec;28(4):379-383.

[30] Asrani SK, Larson JJ, Yawn B, Therneau TM, Kim WR. Underestimation of liverrelated mortality in the United States. Gastroenterology 2013 Aug;145(2):375-382. 